\title{
High expression of RABL6 promotes cell proliferation and predicts poor prognosis in esophageal squamous cell carcinoma
}

\author{
Yanfen Feng ${ }^{1,2 \dagger}$, Shumei Yan ${ }^{1,2 \dagger}$, Yuhua Huang ${ }^{1,2 \dagger}$, Qitao Huang ${ }^{1,2}$, Fang Wang ${ }^{1,3^{*}}$ and Yiyan Lei ${ }^{4^{*}}$ (D)
}

\begin{abstract}
Background: Esophageal squamous cell carcinoma (ESCC) is a common malignant carcinoma of digestive system with high mortality. RAB, member RAS oncogene family like 6 (RABL6), a member of the RAS subfamily, has been reported as an important molecule in several cancers. However, its potential role in ESCC still remains unclear.

Methods: RABL6 mRNA expression was detected in 93 frozen ESCC samples using quantitative reverse transcription-polymerase chain reaction (qRT-PCR). Immunohistochemistry was applied to evaluate the RABL6 expression in tissue microarray containing 171 pairs of ESCC tissues and paired para-cancerous tissues. We evaluated RABL6 expression and its correlation with clinicopathological characteristics and survival. Subsequently, the impact of RABL6 knockdown on the ability of cell proliferation, apoptosis, migration and epithelial-mesenchymal transition (EMT) of ESCC cells was investigated by MTS, Focus formation, flow cytometry, Transwell assays, GRT-PCR, western blot, inverted microscope observation and phalloidin staining, respectively.

Results: Compared to paired para-cancerous tissues, RABL6 was highly expressed in ESCC. The RABL6 highexpression was associated with worse prognosis. We also revealed silencing of RABL6 caused inhibition of cell proliferation, invasion and migration. Further experiments demonstrated that knockdown of RABL6 suppressed the aggressive biological activities of ESCC by suppressing EMT in ESCC cells.
\end{abstract}

Conclusions: RABL6 functions as a tumor oncogene in ESCC. It would be a potential biomarker predicting prognosis, and a novelty target for ESCC therapy.

Keywords: Esophageal squamous cell carcinoma, RABL6, Proliferation, Prognosis

\section{Background}

Esophageal cancer (EC) is one of the most common malignant carcinomas worldwide. It ranks the seventh in morbidity and sixth in mortality respectively overall

\footnotetext{
* Correspondence: wangfang@sysucc.org.cn; leiyyzsu@126.com

†Yanfen Feng, Shumei Yan and Yuhua Huang contributed equally to this work.

'State Key Laboratory of Oncology in South China, Collaborative Innovation Center for Cancer Medicine, Sun Yat-sen University Cancer Center,

Guangzhou 510060, Guangdong, China

${ }^{4}$ Department of Thoracic Surgery, the First Affiliated Hospital, Sun Yat-sen University, Guangzhou, Guangdong 510080, People's Republic of China Full list of author information is available at the end of the article
}

global [1]. It is estimated that $4,779,000$ new cases were diagnosed in China in 2015, while 176, 650 new cases in USA in 2019 [2, 3]. EC is also the fifth leading causes of deaths due to cancers in China [2]. For histological subtype, over $90 \%$ of EC cases are squamous cell carcinoma (ESCC). Although advances have been made in multiple therapeutic approaches, including surgery, radiotherapy, chemotherapy and combination therapy, the prognosis is still dissatisfactory. Currently, the exact oncogenic molecular mechanisms of ESCC remain unclarified. And effective prognostic biomarkers haven't been found yet.

C C The Author(s). 2020 Open Access This article is licensed under a Creative Commons Attribution 4.0 International License, which permits use, sharing, adaptation, distribution and reproduction in any medium or format, as long as you give appropriate credit to the original author(s) and the source, provide a link to the Creative Commons licence, and indicate if changes were made. The images or other third party material in this article are included in the article's Creative Commons licence, unless indicated otherwise in a credit line to the material. If material is not included in the article's Creative Commons licence and your intended use is not permitted by statutory regulation or exceeds the permitted use, you will need to obtain permission directly from the copyright holder. To view a copy of this licence, visit http://creativecommons.org/licenses/by/4.0/ The Creative Commons Public Domain Dedication waiver (http://creativecommons.org/publicdomain/zero/1.0/) applies to the data made available in this article, unless otherwise stated in a credit line to the data. 
Herein, discovering new prognostic predicting marker is important, especially in personalized treatment era.

RABL6 is a novel gene, also known as chromosome 9 open reading frame 86 (C9orf86), or Rab-like protein 1 (RBEL1), or partner of alternative reading frame protein (PARF). Data from the National Center for Biotechnology Information (NCBI) shows that it located at $9 \mathrm{q} 34.3$ [4]. It is reported that $R A B L 6$ is a member of the Ras subfamily which are made up of small GTPases. The GTPases have important effect in various cellular functions, such as cell proliferation, differentiation, survival, and so on [5]. Recently, non-small cell lung cancer (NSCLC), breast cancer and pancreatic ductal adenocarcinoma have been showed to overexpress RABL6, and that was closely correlated with poor prognosis [6-9]. In breast cancer cell lines SK-BR-3 and MCF-7, silencing of $R A B L 6$ by siRNA suppressed cell proliferation and invasion capabilities in vitro [7]. Similarly, knocking down of RABL6 in osteosarcoma cells also impaired cell colony formation and proliferation [10]. These data implicated that $R A B L 6$ is probably a potential oncogene and therapeutic target in cancers.

However, the role of RABL6 in ESCC has not been studied so far. Herein, we carried out this study, to explore the correlation of RABL6 expression and the clinicopathological characteristic as well as prognosis. Furthermore, the function of RABL6 in the tumorigenesis of ESCC was studied by silencing of RABL6 in vitro.

\section{Methods}

\section{Patients and sample collection}

In this study, 171 ESCC cases, who received treatment of esophagectomy in the department of thoracic surgery, Sun Yat-sen university cancer center (SYSUCC) (Guangzhou, China) from November 2000 to November 2007, were enrolled. Histological diagnosis was confirmed by pathologists. Patients who have accepted preoperative chemotherapy or radiation, or had other malignant tumors were excluded. We collected patients' information about their clinical data and pathological characteristics from patients' medical records. We defined Overall survival (OS) as the date on which patients underwent surgery to the date on which patients been last followed, or death due to any cause. The ethics committee of SYSUCC approved this study.

\section{Immunohistochemistry (IHC)}

IHC staining was performed with a tissue microarray which contained two cancer tissues and one matched esophageal normal tissue of 171 cases of ESCC, to measure the expression of RABL6. Primary antibody was by use of a mouse monoclonal antibody against RABL6 (No.400055684-A01, with a dilution of 1:1200, Abnova). Positive control was using a slide with known immuno- reactivity with RABL6, while negative control was using normal rabbit serum. Two pathologists reviewed the IHC slides, counted positive tumor cell percentage in five representative fields to evaluate the expression of RABL6 expression and determine the IHC scores independently. The patients' clinical characteristics was not displayed to the pathologists. Staining extent was scored basing on the proportion of cells with immuno-reactivity in a microscope field: $0,0-10 \%$ of cells stained; $1,10-$ $25 \%$ of cells stained; $2,26-50 \%$ of cells stained; $3,51-$ $75 \%$ of cells stained; 4, 76-100\% of cells stained. And staining intensity varying from weak to strong marked as different scores: negative marked 0, light yellow marked 1 , heavy yellow marked 2, and brown marked 3. Any disagreement of immunohistochemical result analysis was discussed and achieved consensus by these two pathologists. If consensus was still not achieved, a third senior pathologist made the decision. The overall score was the result of staining extent score multiplied by staining intensity score. High expression was defined as scores higher than or equal to median score, and low expression was defined as scores less than median score.

\section{Cell culture and transfection}

The NE1 immortalized esophageal epithelial cell line was obtained from Professor Libing Song from SYSUCC, while the Het-1A cell line was applied by the American Type Culture Collection (ATCC, Manassas, VA, United States). ESCC cell line EC9706 were obtained from TOKU-E Company (Bellingham, WA, United States); Leibniz-Institut DSMZ (Braunschweig, Germany) supplied cell lines KYSE30, KYSE150, KYSE180 and KYSE510; TE-1, TE-9, TE-2, TE-11 and TE-5 were purchased from the RIKEN BRC Cell Bank; Deutsche Sammlung von Mikroorganismen und Zellkulturen (DSMZ, Braunschweig, Germany) supplied cell lines KYSE520 and HK1. The NE1 and Het-1A cells were stored in mixed serum-free medium and EpiLife medium (Invitrogen, Carlsbad, CA, United States); while all cell lines were authenticated before used according to STR fingerprinting as described previously [11]. RABL6 knockdown was accomplished by use of small interfering RNA (siRNA). RNA interference siRNA oligonucleotides and non-targeting siRNA were purchased from $\mathrm{OBiO}$ Company (Shanghai, China). We seeded ESCC cells in dishes with 6 wells. And $2 \times 10^{5}$ cells were seeded per well. Twenty-four hours after seeding, knockdown experiments were done. Cells were transfected by using $\mathrm{Li}$ pofectamine 2000, supplied by Invitrogen (Carlsbad, CA, United States). Following the instructions of manufacturer, we transfected cells with 50 nanomoles negative control siRNA (NC) or two RABL6 siRNA (RABL6siRNA) duplex oligonucleotides, RABL6-siRNA\#1: GGCCTAAAGTACCTTCATA; and RABL6-siRNA\#2: 
GTCATGATGTTCGACATTA. Based on the WB results of RABL6 expression by ESCC cell lines, TE-2 and YES-2 were not the highest expression cell lines, however we observed the most significant morphology changes after RABL6 knockdown in these two cell lines among all cell lines we tested. So, we chose YES2 and TE2 cell lines to perform downstream experiments.

\section{Cell proliferation}

MTS assay (Promega, Madison, WI, United States) was used to evaluate cell proliferation. We carried out the assay according to the experimental protocol described previously [11]. Briefly, 1500 cells were seeded into a plate with 96 wells plates with $200 \mu \mathrm{L}$ media, and cultured to the specified days. Then $20 \mu \mathrm{L}$ MTS solution was added in the plates incubated for another $2 \mathrm{~h}$. Finally, by use of an enzymatic-reader (Thermo Scientific, Waltham, MA, United States), each well's optical density was measured at $490 \mathrm{~nm}$. We repeated independent experiments for 3 times. The data were expressed as Mean \pm Standard Error of Mean (SEM).

\section{Focus formation}

Focus formation was performed as described previously [11]. Briefly, in a plate with 6 wells, we plated 500 cells inside. After 10 days, we fixed surviving colonies, stained them by using crystal violet staining and counted cell colonies. Independent experiments were done triple times, and the data were showed in the form of Mean \pm SEM.

\section{Transwell migration and invasion assays}

Transwell chambers inserts for 24-well plates were supplied by Corning Incorporated (New York, United States). Briefly, in the upper chamber, $1 \times 10^{5}$ cells/ well in $200 \mu \mathrm{L}$ medium were seeded. And in the lower chamber, we added $600 \mu \mathrm{L}$ of medium (with $80 \%$ FBS) to conduct a chemoattractant. Cells were incubated for $24 \mathrm{~h}$ at $37^{\circ} \mathrm{C}$. And then a cotton swab was used to remove the remaining cells on the surface of the upper. Cells migrated to the bottom of the filters were counted under microscope. Before counting, the cells were fixed by $4 \%$ formaldehyde and stained with $0.5 \%$ crystal violet. And then we counted the cells in five photographed fields.

\section{Apoptotic analysis}

Annexin V-FITC/PI Apoptosis Detection Kit (BD Biosciences, Franklin Lakes, United States) was used to detect apoptotic cells according to instruction of manufacturer. After transfection, the cells were treated by with Annixin $\mathrm{V}$ and PI, and examined by the Flow cytometry (BD Biosciences, San Diego, United States).

\section{RNA extraction and qRT-PCR}

The methods of RNA extraction and qRT-PCR were conducted as what was reported previously in the literature [11]. We extracted total RNA and conducted reverse transcription by using Trizol Reagent and Superscript III Reverse Transcriptase (Invitrogen, Carlsbad, CA, United States) respectively. We listed the primer sequences in the Supporting Materials. And the reactions were performed thrice with ABI PRISM 7900 Sequence Detector, with a SYBR Green PCR Kit (Supplied by Biosystems, Carlsbad, CA, United States). The relative expression levels were quantified and analyzed by the SDS 2.3 software (Applied Biosystems, Foster City, CA, United States). GADPH was applied as an endogenous reference.

\section{Western blot analysis}

The protocol of western blot assay were performed as reported previous [11]. The dilution of RABL6 (No.400055684-A01), E-cadherin (ab15148), $\beta$-catenin (ab16051), Vimentin (ab8978) and slug (ab106077) used for western blot was 1:1000, while the dilution of atubulin and GADPH was 1:5000. $\beta$-catenin, slug, atubulin (ab7291), GADPH (ab181602), E-cadherin and Vimentin was applied by Cell Signaling Technology (Beverly, MA, United States). GADPH and a-tubulin was probed on the membranes as an internal control antibody, for the sake of confirming equal loading.

\section{Morphological observation and phalloidin staining}

Cell morphology changes of ESCC with or without knocking down of RABL6 were observed by inverted microscope, and representative images were taken. For phalloidin staining, cells growing on the glass slide were fixed in $4 \%$ formaldehyde at room temperature for half an hour, and then we rinsed the slides 3 times with PBS. After that, phalloidin-conjugate working solution (Phalloidin-iFluor 555 Reagent, ab176756) was added on the slides and incubated at room temperature for an hour. And we rinsed all slides 3 times with PBS. DAPI (blue) was applied to stain nuclear DNA. Finally, we observed cell morphology change under the fluorescence microscope, and took representative images.

\section{Statistical analysis}

The data were recorded as Mean \pm SEM and analyzed through Graphpad prism software (San Diego, CA, United States). One-way analysis of variance (ANOVA) and Newman Keul's multiple comparison tests were applied to analyze the significant differences of more than two groups. The expression of RABL6 between tumor samples and matched non-cancerous samples was analyzed by paired t-test. Survival data were analyzed through Kaplan-Meier survival curves. Independent 
prognostic factors were indentified by cox proportional hazards regression model. $P$ value $<0.05$ was set as statistically significant.

\section{Results}

RABL6 was overexpressed by ESCC tissues and predicted worse prognosis

The expression levels of $R A B L 6$ were compared between normal esophageal epithelial cell line NE-1 and Het-1A and all tested cancer cell lines by qRT-PCR. The average fold change of RABL6 mRNA was obviously higher in all tested cancer cell lines compared with NE-1 and Het1A, except KYSE150 (Fig. 1a). Compared with Het-1A, western blot analysis showed that RABL6 expression was higher in cancer cell lines (Fig. 1b). To compare the expression of RABL6, two independent sets of human samples were used. Fresh tissues of 93 ESCC cases and paired non-cancerous tissues were tested by qRT-PCR; while an ESCC tissue microarray (including 171 informative pairs of cancerous and corresponding noncancerous tissues) were tested by IHC staining with a monoclonal RABL6 antibody, respectively. QRT-PCR results demonstrated that in cancerous tissues, the average fold change of RABL6 mRNA was obviously higher than those in paired non-cancerous and prognosis of ESCC patients was analyzed statistically. For the IHC staining results, the median score was set at 5 . According to the final scores, we divided all cases into two groups: the high RABL6 expression group, with the scores $\geq 5$, and the low RABL6 expression group, with the scores $<5$. The median overall survival rate (OS) and median disease-free survival rate (DFS) of all cases with RABL6 high-expression was significantly lower than those with low-expression (44.4 months vs. 70.4 months, $P=0.001$ and 39.7 months vs. 94.0 months, $P=0.001$, respectively) by Kaplan-Meier analysis. Multivariate analysis indicated that RABL6 was an independent prognostic factor in this cohort of 171 resected ESCC patients (HR $=1.631$; 95\% CI, 1.03-2.59; $P=0.038$, Table 1).

\section{RABL6 expression was obviously correlated with lymph- vascular invasion}

Clinical parameters of the 171 ESCC patients, including age, gender, tumor location, lymph-vascular invasion, peri-neural invasion, histological differentiation, pathological stage and lymph node metastatic status are summarized in Table 2. The location of tumor was defined as upper esophagus, middle esophagus, and lower esophagus, according to where the tumors located. Pathological stage was defined according to the criteria

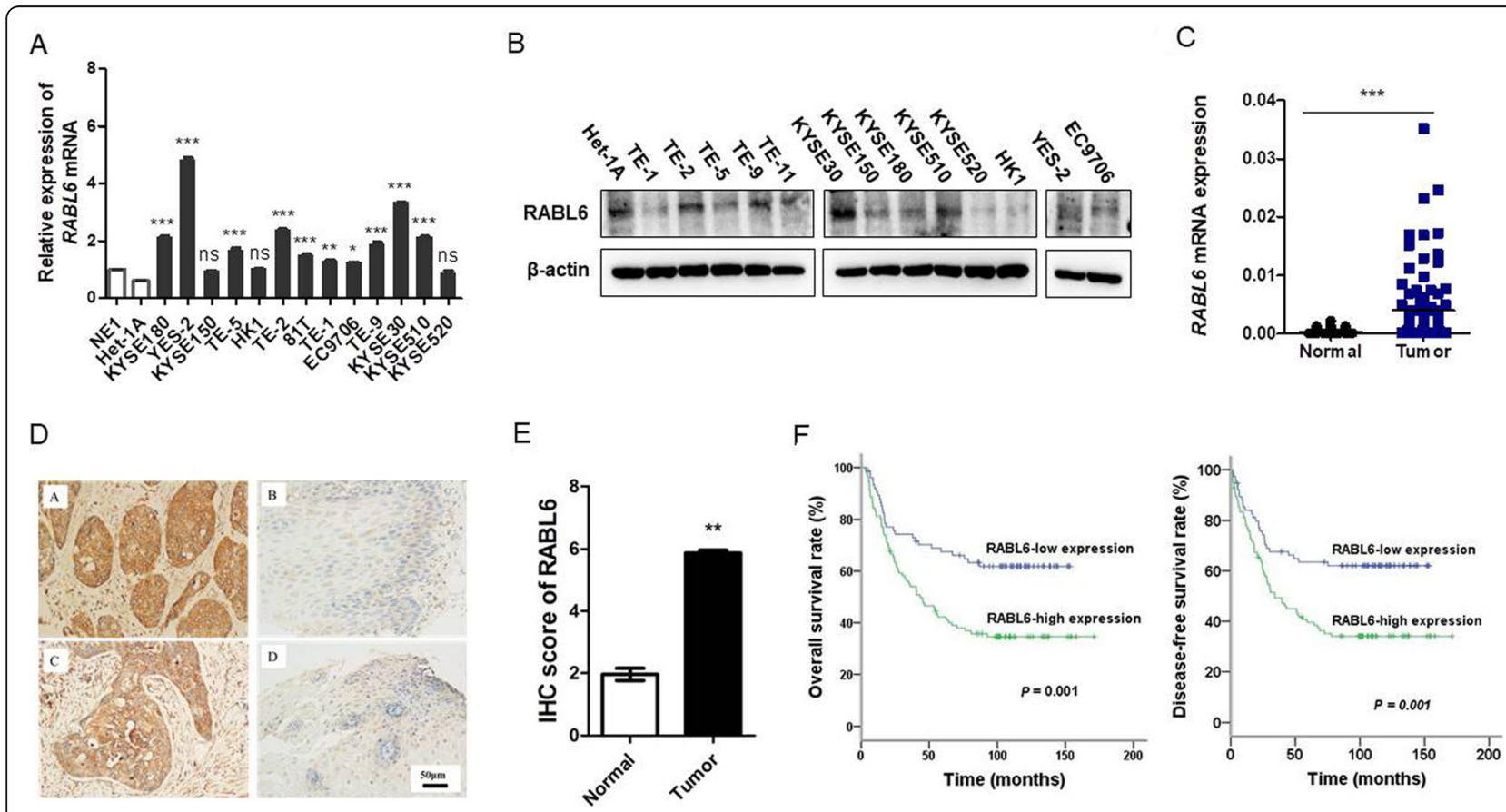

Fig. 1 RABL6 is highly-expressed by ESCC and is associated with worse prognosis. a RABL6 expression was tested and compared between normal esophageal epithelial cell line NE-1 and Het-1 and all tested ESCC cell lines via qRT-PCR. b Expression of RABL6 in different ESCC cell lines and in normal esophageal epithelial cell line Het-1A was compared by Western blot analysis; the original full-length gels are presented in Supplementary Figure S1. c-d The scores of RABL6 expression in 171 ESCC cancerous samples were compared with matched non-cancerous samples by immunohistochemistry staining. c Representative images of RABL6 IHC staining in 2 pairs of ESCC cases (original magnification: 100x). d Results are expressed in the form of mean+/-SEM. e- $\mathbf{f}$ Kaplan-Meier analysis showed the overall survival rate and disease-free survival rate of ESCC patients stratified by $R A B L 6$ expression. * indicates $P<0.05$, ${ }^{* *}$ indicates $P<0.01$, ** indicates $P<0.01$ for statistical results 
Table 1 Univariate and multivariate Cox Regression analyzes for overall survival (OS) in ESCC patients

\begin{tabular}{|c|c|c|c|c|}
\hline Factors & HR $(95 \% \mathrm{Cl})$ & $P$ value & HR $(95 \% \mathrm{Cl})$ & $P$ value \\
\hline Age ( $\leq 55$ vs. $>55)$ & $0.956(0.63-1.45)$ & 0.833 & - & - \\
\hline Gender & $0.468(0.27-0.83)$ & 0.009 & $0.608(0.34-1.08)$ & 0.092 \\
\hline Tumor location & $0.813(0.55-1.19)$ & 0.293 & - & - \\
\hline Lymph-vascular invasion & $0.479(0.31-0.73)$ & 0.001 & $0.479(0.31-0.73)$ & 0.001 \\
\hline Perineural invasion & $0.652(0.43-0.99)$ & 0.044 & $0.766(0.49-1.19)$ & 0.243 \\
\hline Histological differentiation & $1.666(1.22-2.27)$ & 0.001 & $1.396(1.02-1.90)$ & 0.035 \\
\hline Pathological Stage & $3.458(2.26-5.29)$ & 0.000 & $2.233(1.09-4.58)$ & 0.028 \\
\hline Lymph node metastasis & $3.650(2.33-5.71)$ & 0.000 & $1.566(0.74-3.31)$ & 0.240 \\
\hline $\begin{array}{l}\text { RABL6 expression } \\
\text { (High vs. low) }\end{array}$ & $2.119(1.35-3.32)$ & 0.001 & $1.631(1.03-2.59)$ & 0.038 \\
\hline
\end{tabular}

HR Hazard ratio, $\mathrm{Cl}$ confidential interval

of the AJCC (2017 version). Patients' median age in this study was 55.0 years (range: $30.0-75.0$ years). There were $129(75.5 \%)$ males and $42(24.6 \%)$ females (male to female ratio, 3.07:1). RABL6 expression was associated with patients' age and lymph-vascular invasion $(P=$ 0.040 and $P<0.000$, respectively). High expression of

Table 2 Baseline characteristics of ESCC patients and the correlation with RABL6 expression

\begin{tabular}{|c|c|c|c|c|}
\hline \multirow[t]{2}{*}{ Characteristics } & \multirow{2}{*}{$\begin{array}{l}\text { Total [cases (\%)] } \\
N=171\end{array}$} & \multicolumn{2}{|c|}{ RABL6 expression [cases (\%)] } & \multirow[t]{2}{*}{$P$ value } \\
\hline & & $\begin{array}{l}\text { High } \\
N=96\end{array}$ & $\begin{array}{l}\text { Low } \\
N=75\end{array}$ & \\
\hline Age (years) & & & & 0.040 \\
\hline$\leq 55$ & $79(46.2)$ & $45(26.3)$ & $34(19.9)$ & \\
\hline$>55$ & $92(53.8)$ & $51(29.8)$ & $41(24.0)$ & \\
\hline Gender & & & & 0.320 \\
\hline Male & $129(75.4)$ & $74(43.2)$ & $55(32.2)$ & \\
\hline Female & $42(24.6)$ & $22(12.9)$ & $20(11.7)$ & \\
\hline Tumor location & & & & 0.132 \\
\hline Upper & $13(7.6)$ & $8(4.7)$ & $5(2.9)$ & \\
\hline Middle & $114(66.7)$ & $69(40.4)$ & $45(26.3)$ & \\
\hline Low & $44(25.7)$ & $19(11.1)$ & $25(14.6)$ & \\
\hline Lymph-vascular invasion & & & & 0.000 \\
\hline Yes & $79(46.2)$ & $56(32.7)$ & $23(13.5)$ & \\
\hline No & $92(53.8)$ & $40(23.4)$ & $52(30.4)$ & \\
\hline Perineural invasion & & & & 0.139 \\
\hline Yes & $63(36.8)$ & $40(23.4)$ & $23(13.5)$ & \\
\hline No & $108(63.2)$ & $56(32.7)$ & $52(30.4)$ & \\
\hline Histological differentiation & & & & 0.282 \\
\hline Well & $39(22.8)$ & $22(12.9)$ & $17(9.9)$ & \\
\hline Moderate & $93(54.4)$ & $48(28.1)$ & $45(26.3)$ & \\
\hline Poor & $39(22.8)$ & $26(15.2)$ & $13(7.6)$ & \\
\hline Pathological stage & & & & 0.374 \\
\hline$|\&| \mid$ & $103(60.2)$ & $55(32.2)$ & $48(28.1)$ & \\
\hline III \& IV & $68(39.8)$ & $41(23.9)$ & $27(15.8)$ & \\
\hline Lymph node metastasis & & & & 0.162 \\
\hline No & $90(52.6)$ & $46(26.9)$ & $44(25.7)$ & \\
\hline Yes & $81(47.4)$ & $50(56.1)$ & $31(18.1)$ & \\
\hline
\end{tabular}


RABL6 was more common in patients older than 55 years, and in patients with lymph-vascular invasion. No statistical association was found between expression of RABL6 and other clinicopathological characteristics such as gender, tumor location, peri-neural invasion, histological differentiation, pathological stage, and lymph node metastatic status in our study (Table 2).

\section{Knockdown of RABL6 inhibited proliferation of cancer cells}

Owing to RABL6 high-expression predicted poorer prognosis in ESCC patients, we raised a hypothesis that RABL6 might play oncogenic roles in ESCC, and RABL6 expression might promote cancer cell growth. So, the role of RABL6 on cell growth was invested via knockdown of RABL6. We used small interfering RNA (siRNA) to knock down RABL6 in YES-2 and TE-2 cells stably. The expression of RABL6 in RABL6-knocked-down cells was examined via qRT-PCR method (Fig. 2a). MTS assay displayed that cell growth rates in RABL6-knocked-down YES2 and TE-2 cells were obviously lower than the negative control cells (NC) (Fig. 2b, c). The result of focus formation assay showed that the RABL6knocked-down YES-2 and TE-2 cells formed lower number and smaller colonies than the control cells (Fig. 2d, e). All the results indicated that knockdown of RABL6 inhibited cell proliferation and growth in YES-2 and TE-2 cells.

\section{Knockdown of RABL6 had no influence on apoptosis in ESCC cells in vitro}

The effect of RABL6 in cell apoptosis was evaluated by flow cytometry analysis. Results revealed that RABL6 silencing couldn't induce apoptosis in YES-2 and TE1 cells (Fig. 3a, b).

\section{Knockdown of RABL6 inhibited migrating and invading of ESCC cells}

Since the statistical results showed that RABL6 expression was closely associated with lymph-vascular invasion, we studied the influence of RABL6 on migration and invasion in ESCC cells through Transwell assay. Results showed that the abilities of migrating and invading of ESCC cells were significantly decreased after silencing of RABL6 (Fig. 4a, b, c, d). These results indicated that knockdown of $R A B L 6$ remarkably inhibited migrating and invading of cancer cells.

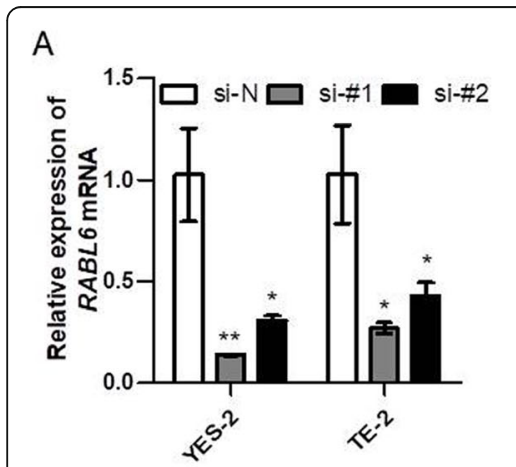

B

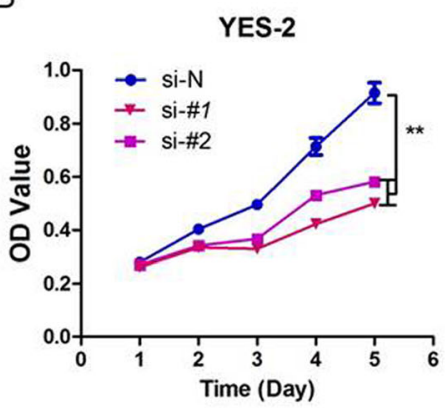

C

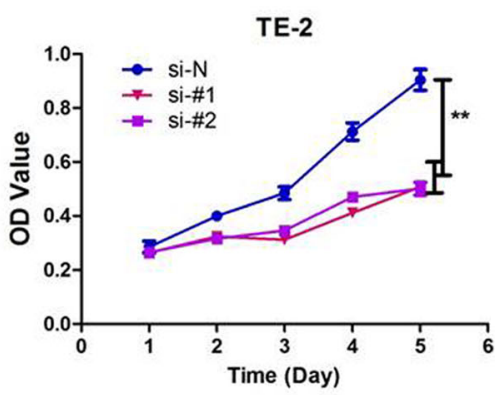

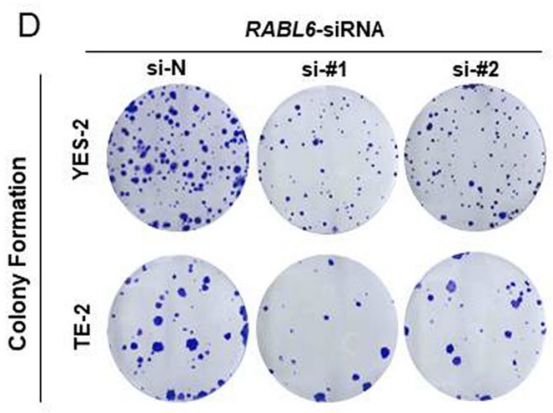

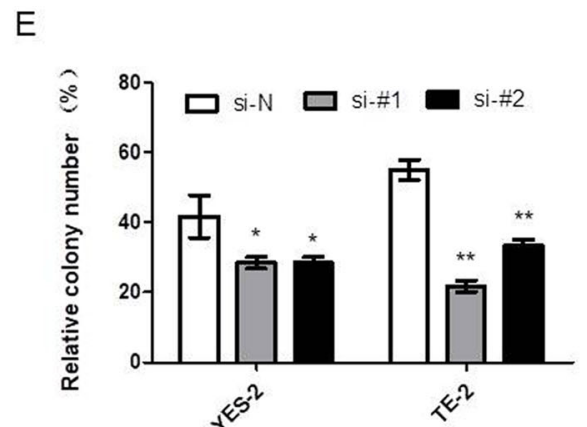

Fig. 2 Knockdown of RABL6 suppressed ESCC cell proliferation. a-e YES-2 and TE-2 cells were transfected with siRNAs specifically targeting RABL6 (si-\#1, si-\#2) or scrambled siRNA control (si-N). a QRT-PCR confirmed the effective knockdown of RABL6 in these ESCC cells. b-c MTS assay tested the cell growth rate of these RABL6 knockdown cells, and the results were analyzed statistically. $\mathbf{d}$-e Single cell clone assay detected the colony formation. $\mathbf{d}$ Representative image of clone formation e Results are analyzed statistically. The experiments were repeated 3 times independently, and the representative data of the experiments are presented in figures. * indicates $P<0.05$, ** indicates $P<0.01$ for statistical results 
A

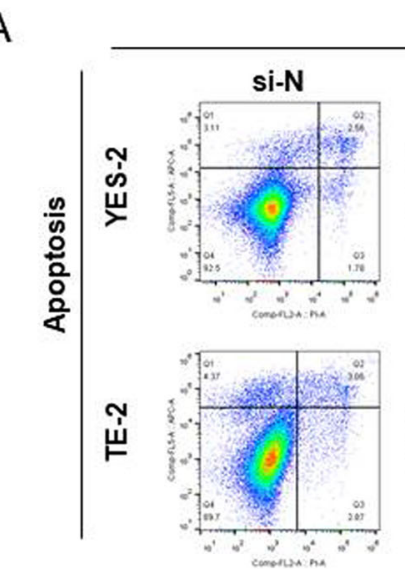

RABL6-siRNA

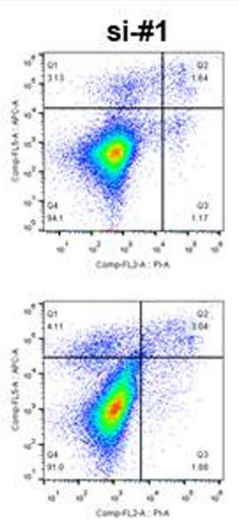

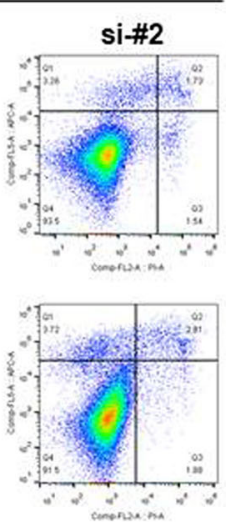

B

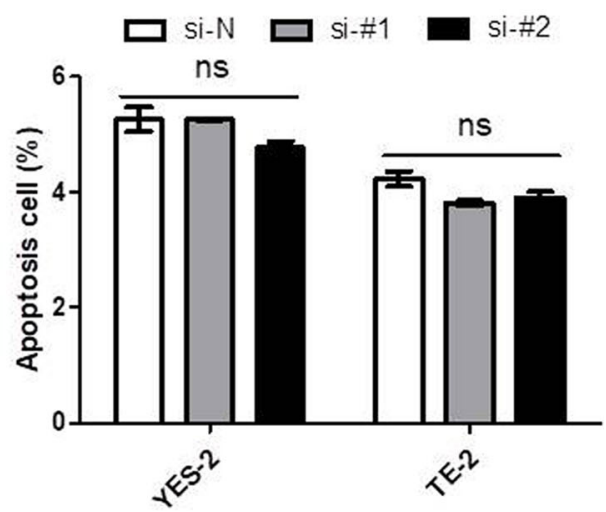

Fig. 3 Knockdown of RABL6 had no impact on ESCC cells. a Flow cytometry assay was performed to analyze apoptosis of YES-2 and TE-2 cells with RABL6 knockdown. b Results are statistically analyzed. Values represented the mean \pm SD data in triplicate, ns: no significance

Knockdown of RABL6 inhibited epithelial-mesenchymal transition (EMT) in ESCC cells

Whether RABL6 promoted tumor cell migration via inducing EMT remains unclear. In order to analyze the function of RABL6 on EMT, we studied the expression of EMT markers and EMT-related transcription factors through qRT-PCR and western blot, and also observed cell morphology changes by inverted microscope in RABL6 knockdown cells and control cells. QRT-PCR results showed that the expression of epithelial markers Ecadherin and $\beta$-catenin was obviously increased, and the mesenchymal marker Vimentin and slug was significantly decreased in knocked-down RABL6 cells compared to control groups (Fig. 5a, b). The results of

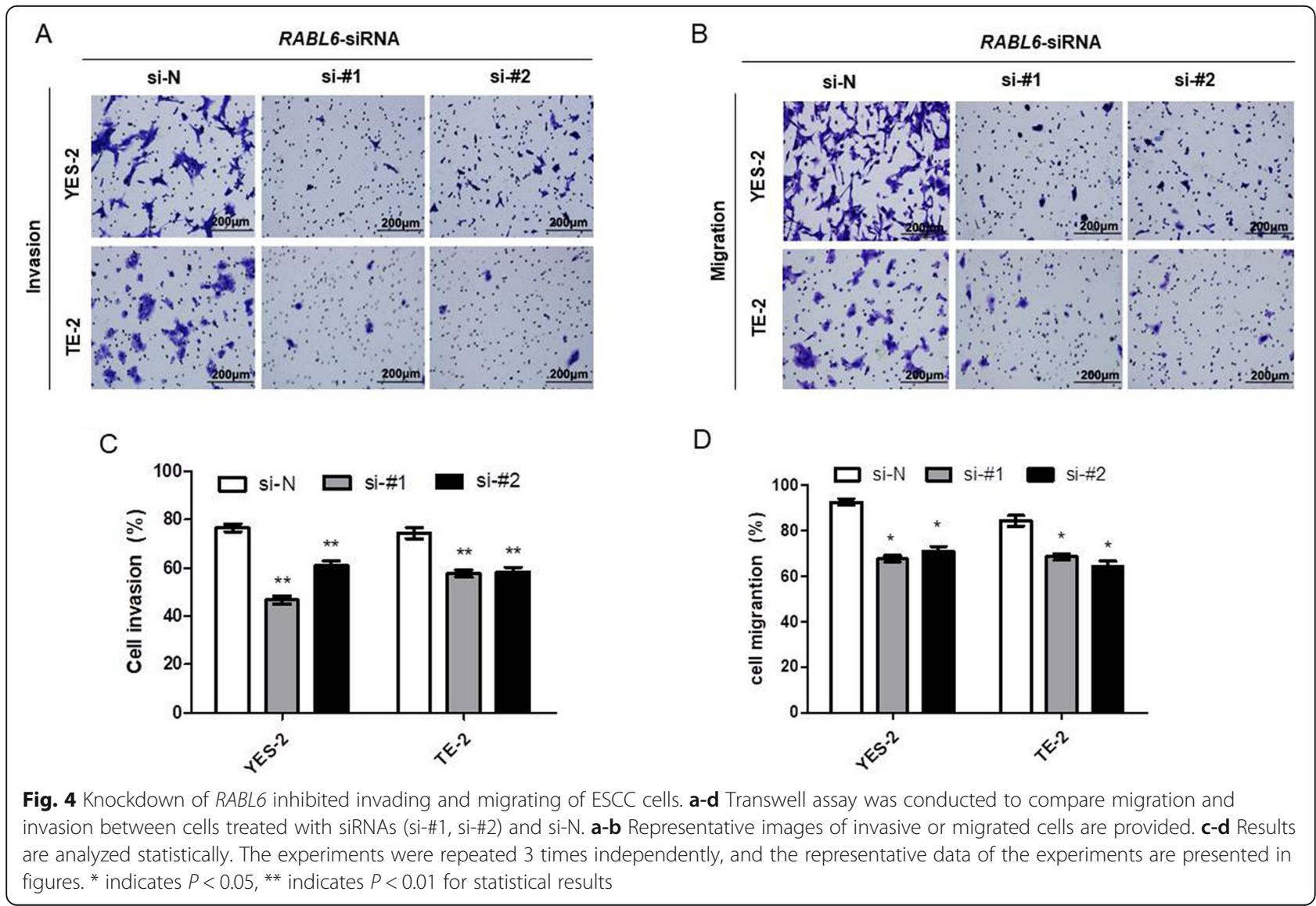



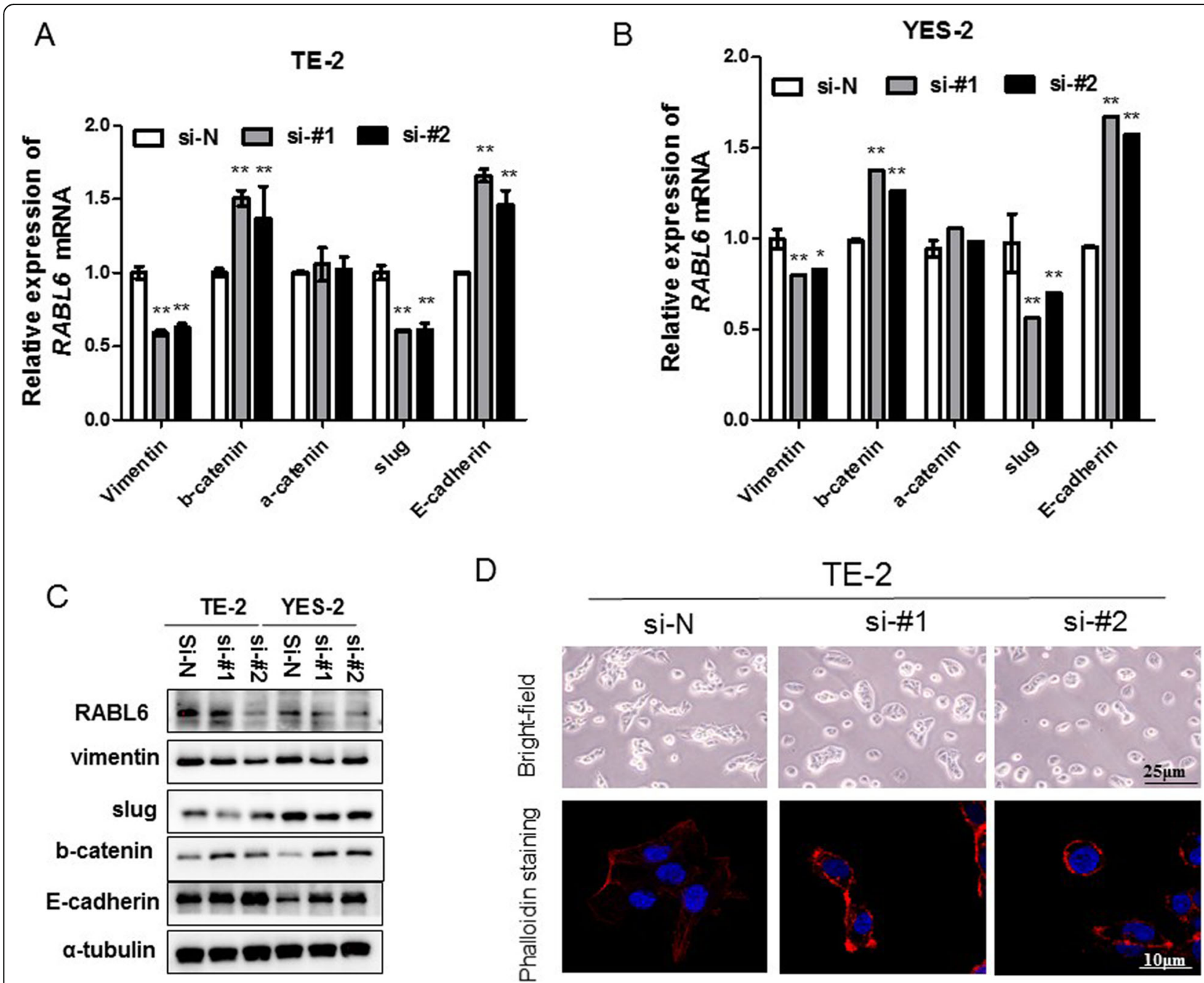

Fig. 5 Knockdown of RABL6 inhibited epithelial-mesenchymal transition in ESCC cells (a-c). a-b Relative expressions of E-cadherin, a-catenin, bcatenin, Vimentin, and Slug in YES-2 and TE-2 cells were compared by qRT-PCR between siRNA RABL6-silenced cells (sii\#-1, si-\#2) and their control cells (si-N). c Western blots comparing siRNA RABL6-silenced cells (sil\#-1, si-\#2) with their control cells (si-N) are showed with the expression of vimentin, slug, $\beta$-catenin and E-cadherin. A-tubulin was taken as control; the original full-length gels are presented in Supplementary Figure S2. d Representative bright-field images of ESCC cells and ESCC cells stained with fluorescent phalloidin to show actin structures, with or without silencing of RABL6. DAPI (blue) was applied to stain nuclear DNA. (original magnification of bright-field: 400x; original magnification of fluorescent phalloidin: 1000x). Data are expressed in the form of the mean \pm SEM. * indicates $P<0.05$, ${ }^{* *}$ indicates $P<0.01$ versus the control

western blot were consistent with the results of qRTPCR (Fig. 5c). We observed by bright field that RABL6 knockdown ESCC cells were less spindle and became cobble stone like shape compared to their negative control cells in shape. Phalloidin staining showed circumferential actin belts in RABL6 knockdown ESCC cells, while the actin structures were spindle-shaped in the control cells (Fig. 5d). This indicated the occurrence of EMT. All the results together revealed that silencing of RABL6 inhibited EMT in ESCC cells.

\section{Discussion}

Esophageal cancer is one of the most common malignant carcinomas worldwide, especially in China. Surgical treatment is a main therapeutic modality for early stage and locally advanced ESCC. For locally advanced cases, neoadjuvant or adjuvant chemotherapies are now applied. For unresectable or late-staged cases, the concurrent chemoradiotherapy maybe the recommended therapy [12]. Nevertheless, the treatment outcome for ESCC is still under satisfactory with these traditional therapeutic methods. Recently, targeted therapy has led to significant breakthroughs in cancer therapy, such as metastatic melanoma, NSCLC, gastrointestinal stromal tumor, and so on [13]. For ESCC, some studies reveal that mTOR, PTEN, and Forkhead box M1 (FOXM1) maybe the prognostic predictors and therapeutic targets $[14,15]$. However, effective targeted therapy for ESCC is 
still needed to be developed, due to the exact cellular and molecular mechanisms of oncogenesis and progression for ESCC remains unclear.

RABL6 is a novel Ras superfamily protein. The Ras superfamily of GTPases comprises several subfamilies of small GTP-binding proteins which play pivotal roles in tumorigenesis, as their functions included cell proliferating, differentiating, and apoptosis [16, 17]. Several studies found elevated RABL6 expression in various human cancers, including pancreatic ductal adenocarcinomas, pancreatic neuroendocrine tumors and breast tumors. And RABL6 overexpression was associated with poor survival in those cancers [7-9]. However, its role in ESCC is yet to be discovered. Here we firstly investigated RABL6 in ESCC. We found that RABL6 was overexpressed in ESCC tissues and cell lines. And patients with high RABL6 expression had statistical poorer prognosis than those with low RABL6 expression. High RABL6 expression was an independent prognostic factor in ESCC. And patients with high expression of RABL6 had obviously higher rate of lymph-vascular invasion compared to those with low expression of RABL6. Studies showed that metastasis and recurrence are key factors contributing to poor prognosis of cancer, and the presence of lymph-vascular invasion strongly associated with high risk of metastasis and recurrence in endometrial cancer, ESCC, and so on $[18,19]$. These may be one of the reasons to explain RABL6 high expression associated with poor prognosis, and implied that $R A B L 6$ played a vital role in the malignant progress of ESCC.

To investigate the role of $R A B L 6$, a series of functional studies were carried out. In vitro studies showed that knockdown of RABL6 inhibited tumor cell growth, proliferation, invasion and migration. Tang et al. found that silencing of RABL6 gene in U2-OS and SAOS2 osteosarcoma cell lines suppressed cell colony formation and proliferation in vitro [10]. They also demonstrated that RABL6 modulated G1-S transition in cell cycle analysis. RABL6 regulated retinoblastoma 1 (Rb1) activity in osteosarcoma cells, which was the major player in cell cycle control [10, 20, 21]. Li Y et al. also reported that silencing of RABL6 promoted breast cancer cell growth was via promoting apoptosis [7]. Besides cell cycle and apoptosis, Montalbano et al. reported that RABL6 knockdown resulted in marked cell growth suppression, which is associated with inhibition of extracellular signal-regulated kinase phosphorylation [22]. These reports indicated that RABL6 displays an oncogenic function by regulating cell cycle or inducing apoptosis, or influencing signal pathway. However, our study showed that silencing of RABL6 couldn't induce apoptosis in ESCC cell in vitro. Whether RABL6 carried out its oncogenic function in ESCC via regulating cell cycle or signaling pathway needs to be further investigated.
We also demonstrated that knockdown of RABL6 inhibited cell invasion and migration via EMT. It was reported that EMT inducers were important for morphogenesis and organogenesis through regulating cell migrating. The abnormal activation ofEMT played a key role in development and metastasis in tumors [23]. Up regulation of mesenchymal markers, down regulation of epithelial markers, abnormal localization of $\beta$-catenin and nuclear expression of Vimentin are some of its characteristics $[24,25]$. In the development of epithelial tumors, cancer cells obtain phenotypes of invasion and motivation through EMT, which results in invasion or metastasis, leading to the death of about $90 \%$ of patients [26].

Taken all these together, we found that RABL6 plays an important part in the tumorigenesis and progression in ESCC.

\section{Conclusions}

Conclusively, we indicated firstly that RABL6 is highly expressed by ESCC and associated with poor prognosis. Its expression is associated with lymph-vascular invasion. Downregulation of RABL6 suppressed proliferation, migration and EMT of ESCC cells. Thus, RABL6 exert an important function on the progression of ESCC, and may be promising prognostic marker and a potential therapeutic target for ESCC. Its underlying mechanism and clinical application need to be further developed.

\section{Supplementary information}

Supplementary information accompanies this paper at https://doi.org/10. 1186/s12885-020-07068-W.

\section{Additional file 1.}

Additional file 2.

Additional file 3.

\section{Abbreviations}

RABL6: RAB, member RAS oncogene family like 6; C9orf86: Chromosome 9 open reading frame 86; ESCC: Esophageal squamous cell carcinoma; qRTPCR: Quantitative reverse transcription-polymerase chain reaction; EMT: Epithelial-mesenchymal transition; EC: Esophageal cancer; RBEL1: Rablike protein 1; PARF: Partner of alternative reading frame protein; NCBI: National Center for Biotechnology Information; NSCLC: Non-small cell lung cancer; OS: Overall survival; ATCC: American Type Culture Collection; siRNA: Small interfering RNA; SDS-PAGE: Sodium dodecyl sulfatepolyacrylamide gel electrophoresis; ANOVA: One-way analysis of variance; NC: Negative control cells; FOXM1: Forkhead box M1; Rb1: Retinoblastoma 1

\section{Acknowledgements}

Not applicable.

\section{Authors' contributions}

YFF, FW and YYL designed the study. YFF, SMY, YHH and QTH performed the experiments. YFF, FW and YYL analyzed, interpreted the data and wrote the article. All authors have approved this submission.

Funding

Not applicable. 


\section{Availability of data and materials}

The data in this study are available from the corresponding author on request.

\section{Ethics approval and consent to participate}

All the patients in this study signed consents for the approval of use of their tissues and information in research. The institutional ethics committee of Sun Yat-sen University cancer center approved this study.

\section{Consent for publication}

Not applicable.

\section{Competing interests}

The authors declare that they have no conflict of interest.

\section{Author details}

${ }^{1}$ State Key Laboratory of Oncology in South China, Collaborative Innovation Center for Cancer Medicine, Sun Yat-sen University Cancer Center, Guangzhou 510060, Guangdong, China. ${ }^{2}$ Department of Pathology, Sun Yat-sen University Cancer Center, Guangzhou 510060, Guangdong, China. ${ }^{3}$ Department of Molecular Diagnostics, Sun Yat-sen University Cancer Center, Guangzhou 510060, China. ${ }^{4}$ Department of Thoracic Surgery, the First Affiliated Hospital, Sun Yat-sen University, Guangzhou, Guangdong 510080, People's Republic of China.

Received: 19 September 2019 Accepted: 15 June 2020

Published online: 29 June 2020

\section{References}

1. Bray F, Ferlay J, Soerjomataram I, Siegel RL, Torre LA, Jemal A. Global cancer statistics 2018: GLOBOCAN estimates of incidence and mortality worldwide for 36 cancers in 185 countries. Ca-a Cancer J Clin. 2018;68(6):394-424.

2. Chen W, Zheng R, Baade P, Zhang S, Zeng H, Bray F, Jemal A, Yu X, He J. Cancer statistics in China, 2015. CA Cancer J Clin. 2016;66(2):115-32.

3. Siegel RL, Miller KD. Cancer statistics, 2019. CA Cancer J Clin. 2019;69(1):734.

4. Zhang XF, Hagen J, Muniz VP, Smith T, Coombs GS, Eischen CM, Mackie DI, Roman DL, Van Rheeden R, Darbro B, Tompkins VS, Quelle DE. RABL6A, a novel RAB-like protein, controls centrosome amplification and chromosome instability in primary fibroblasts. PLoS One. 2013;8(11):e80228.

5. Wennerberg K, Rossman KL, Der CJ. The Ras superfamily at a glance. J Cell Sci. 2005;118(Pt 5):843-6.

6. Cheng KW, Lahad JP, Kuo WL, Lapuk A, Yamada K, Auersperg N, Liu J, Smith-McCune K, Lu KH, Fishman D, et al. The RAB25 small GTPase determines aggressiveness of ovarian and breast cancers. Nat Med. 2004; 10(11):1251-6.

7. Li YY, Fu S, Wang XP, Wang HY, Zeng MS, Shao JY. Down-regulation of c9orf86 in human breast cancer cells inhibits cell proliferation, invasion and tumor growth and correlates with survival of breast cancer patients. PLoS One. 2013:8(8):e71764.

8. Muniz VP, Askeland RW, Zhang X, Reed SM, Tompkins VS, Hagen J, McDowell BD, Button A, Smith BJ, Weydert JA, et al. RABL6A promotes Oxaliplatin resistance in tumor cells and is a new marker of survival for resected pancreatic ductal adenocarcinoma patients. Genes Cancer. 2013; 4(7-8):273-84

9. Yoshimura K, Osman M, Inoue $Y$, Suda T, Sugimura $H$. A novel prognostic marker of non-small cell lung cancer: chromosome 9 open reading frame 86 (C9orf86). J Thorac Dis. 2016;8(9):2284-6.

10. Tang H, Ji F, Sun J, Xie $Y, X u Y, Y$, $H$. RBEL1 is required for osteosarcoma cell proliferation via inhibiting retinoblastoma 1. Mol Med Rep. 2016;13(2): 1275-80.

11. Feng YF, Lei YY, Lu JB, Xi SY, Zhang Y, Huang QT, Wu QL, Wang F. RIT1 suppresses esophageal squamous cell carcinoma growth and metastasis and predicts good prognosis. Cell Death Dis. 2018;9(11):1085

12. Kato H, Nakajima M. Treatments for esophageal cancer: a review. Gen Thorac Cardiovasc Surg. 2013;61(6):330-5

13. Patel S. Long-term efficacy of imatinib for treatment of metastatic GIST. Cancer Chemother Pharmacol. 2013;72(2):277-86.

14. Lu J, Pan Y, Xia X, Gu Y, Lei Y. Prognostic significance of mTOR and PTEN in patients with esophageal squamous cell carcinoma. Biomed Res Int. 2015; 2015:417210.
15. Song $L$, Wang $X$, Feng Z. Overexpression of FOXM1 as a target for malignant progression of esophageal squamous cell carcinoma. Oncol Lett. 2018;15(4):5910-4.

16. Hernandez-Alcoceba R, del Peso L, Lacal JC. The Ras family of GTPases in cancer cell invasion. Cell Mol Life Sciences. 2000;57(1):65-76.

17. Peng GL, Tao YL, Wu QN, Zhang Y, He JX. Positive expression of protein chromosome 9 open reading frame 86 (C9orf86) correlated with poor prognosis in non-small cell lung cancer patients. J Thorac Dis. 2016;8(7): 1449-59.

18. Loizzi V, Cormio G, Lorusso M, Latorre D, Falagario M, Demitri P, Scardigno $D$, Selvaggi LE. The impact of lymph vascular space invasion on recurrence and survival in patients with early stage endometrial cancer. Eur J Cancer Care. 2014;23(3):380-4.

19. Qu Y, Lin H, Zhang C, Li K, Zhang H. Cribriform pattern in lung invasive adenocarcinoma correlates with poor prognosis in a Chinese cohort. Pathol Res Pract. 2019;215(2):347-53.

20. Burkhart DL, Sage J. Cellular mechanisms of tumour suppression by the retinoblastoma gene. Nat Rev Cancer. 2008;8(9):671-82.

21. Classon M, Harlow E. The retinoblastoma tumour suppressor in development and cancer. Nat Rev Cancer. 2002;2(12):910-7.

22. Montalbano J, Lui K, Sheikh MS, Huang Y. Identification and characterization of RBEL1 subfamily of GTPases in the Ras superfamily involved in cell growth regulation. J Biol Chem. 2009;284(27):18129-42

23. Ansieau S, Courtois-Cox S, Morel AP, Puisieux A. Failsafe program escape and EMT: a deleterious partnership. Semin Cancer Biol. 2011;21(6):392-6.

24. Kanai Y, Oda T, Tsuda H, Ochiai A, Hirohashi S. Point mutation of the Ecadherin gene in invasive lobular carcinoma of the breast. Japanese J Cancer Res. 1994;85(10):1035-9.

25. Gamallo C, Palacios J, Suarez A, Pizarro A, Navarro P, Quintanilla M, Cano A. Correlation of E-cadherin expression with differentiation grade and histological type in breast carcinoma. Am J Pathol. 1993;142(4):987-93.

26. Pradella D, Naro C, Sette C, Ghigna C. EMT and stemness: flexible processes tuned by alternative splicing in development and cancer progression. Mol Cancer. 2017;16(1):8

\section{Publisher's Note}

Springer Nature remains neutral with regard to jurisdictional claims in published maps and institutional affiliations.

Ready to submit your research? Choose BMC and benefit from:

- fast, convenient online submission

- thorough peer review by experienced researchers in your field

- rapid publication on acceptance

- support for research data, including large and complex data types

- gold Open Access which fosters wider collaboration and increased citations

- maximum visibility for your research: over $100 \mathrm{M}$ website views per year

At $\mathrm{BMC}$, research is always in progress.

Learn more biomedcentral.com/submissions 